Provided for non-commercial research and education use. Not for reproduction, distribution or commercial use.

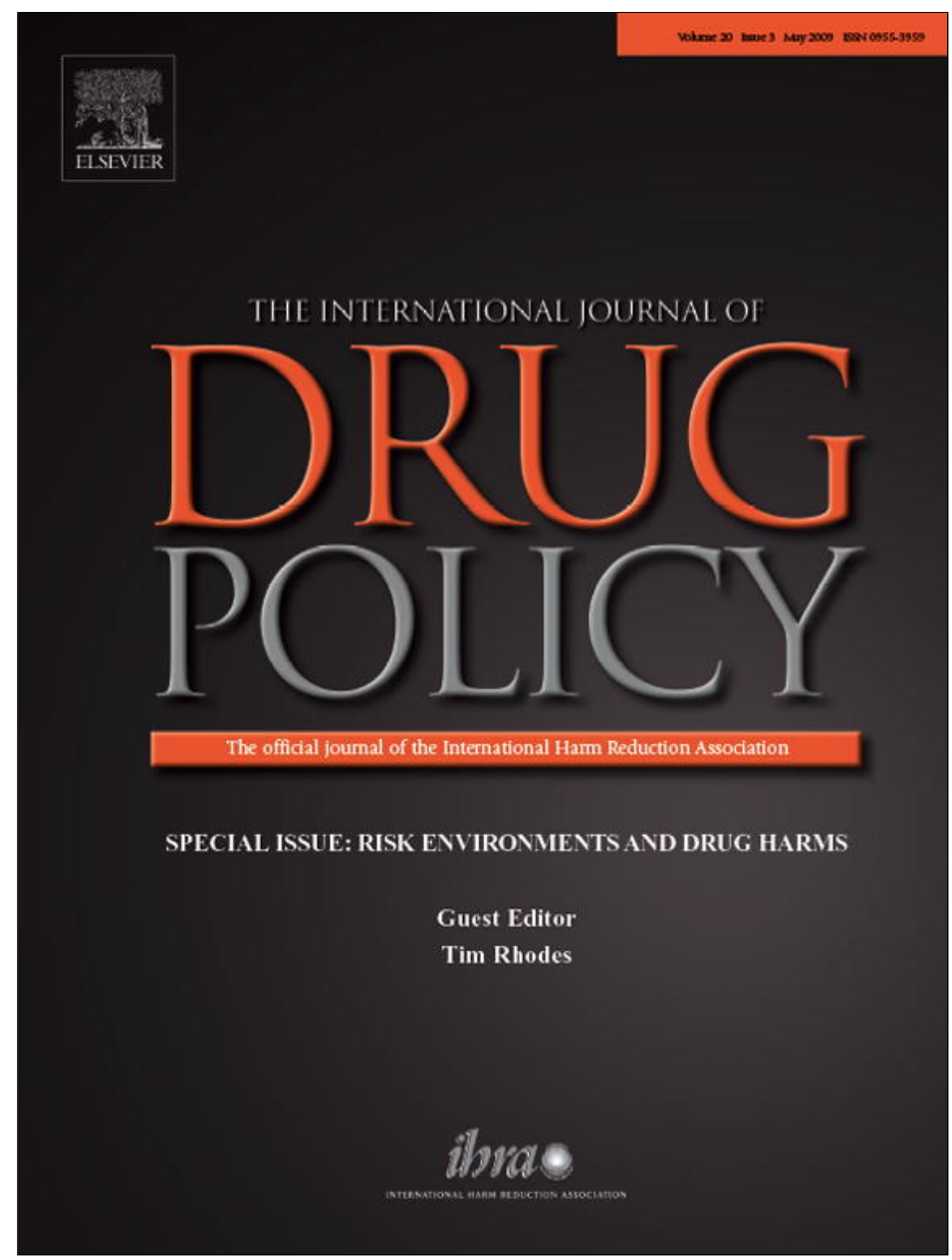

This article appeared in a journal published by Elsevier. The attached copy is furnished to the author for internal non-commercial research and education use, including for instruction at the authors institution and sharing with colleagues.

Other uses, including reproduction and distribution, or selling or licensing copies, or posting to personal, institutional or third party websites are prohibited.

In most cases authors are permitted to post their version of the article (e.g. in Word or Tex form) to their personal website or institutional repository. Authors requiring further information regarding Elsevier's archiving and manuscript policies are encouraged to visit:

http://www.elsevier.com/copyright 


\title{
Commentary
}

\section{Social epidemiology and complex system dynamic modelling as applied to health behaviour and drug use research}

\author{
Sandro Galea*, Chris Hall, George A. Kaplan \\ Center for Social Epidemiology and Population Health, Department of Epidemiology, School of Public Health, University of Michigan, Ann Arbor, MI, United States
}

\section{A R T I C L E I N F O}

\section{Article history:}

Received 3 May 2008

Received in revised form 23 July 2008

Accepted 9 August 2008

\section{Keywords:}

Population health

Multilevel

Regression modeling

Social epidemiology

\begin{abstract}
A B S T R A C T
A social epidemiologic perspective considers factors at multiple levels of influence (e.g., social networks, neighbourhoods, states) that may individually or jointly affect health and health behaviour. This provides a useful lens through which to understand the production of health behaviours in general, and drug use in particular. However, the analytic models that are commonly applied in population health sciences limit the inference we are able to draw about the determination of health behaviour by factors, likely interrelated, across levels of influence. Complex system dynamic modelling techniques may be useful in enabling the adoption of a social epidemiologic approach in health behaviour and drug use research. We provide an example of a model that aims to incorporate factors at multiple levels of influence in understanding drug dependence. We conclude with suggestions about future directions in the field and how such models may serve as virtual laboratories for policy experiments aimed at improving health behaviour.
\end{abstract}

(c) 2008 Elsevier B.V. All rights reserved.

\section{Introduction}

It is abundantly clear that the explanation and prediction of health-related behaviour is tremendously difficult. Substantial theoretic and empiric work has been dedicated to conceptualizing and attempting to analyse the factors that determine health behaviour (Cummings, Becker, \& Maile, 1980; Fishbein et al., 2001; Glantz, Lewis, \& Rimer, 1997). Despite these efforts, many aspects of health-related behaviour remain unexplained. For example, over the past few decades, there have been several instances of dramatic population-based changes in health-related behaviour that were unforeseen by the public health community at large and only occasioned substantial examination after they had become well established. Two recent examples of this are the rise in crack cocaine use in urban areas in the 1980s, and the dramatic rise in overweight and obesity in the 1990s. Informed by this understanding, in this paper we discuss (a) a social epidemiologic, multilevel perspective on health-related behaviour, and (b) how complex system dynamic models may provide an analytic tool to improve our understanding of how factors across levels of influence, and the pathways that link these levels, determine health behaviour. We then present an illustration of how

\footnotetext{
* Corresponding author at: Department of Epidemiology, University of Michigan School of Public Health, 109 Observatory Street, Room 3663, Ann Arbor, MI 481092029, United States. Tel.: +1 734647 9741; fax: +1 7347635706 .

E-mail address: sgalea@umich.edu (S. Galea).
}

complex systems dynamic models might be applied to the understanding of drug dependence. We conclude with some thoughts about potential areas of research and potential directions in the field.

\section{A social epidemiologic perspective on health behaviour and drug use}

There has been a tremendous increase in social epidemiologic inquiry about the determination of health and health behaviour over the past two decades. Although there is no single consensus about what demarcates "social" epidemiology from other areas of epidemiologic inquiry, for the purposes of this discussion we consider social epidemiology to be the area of research concerned with understanding how exogenous factors, including characteristics of individuals' interactions with one another and their environment, influence the occurrence of health and disease, and their associated risk factors. For example, social epidemiologic inquiry in the area of drug use has focused on, among other domains, how social networks influence the incidence and cessation of drug use behaviours (Sussman \& Dent, 1999), how features of the built environment determine the consequences of drug use (Hembree et al., 2005), and how macro-social factors like segregation influence the rates of injection drug use (Cooper, Friedman, Tempalski, \& Friedman, 2007) [For a comprehensive review, see Galea, Nandi, \& Vlahov, 2004; Glass \& McAtee, 2006]. In many respects, inquiry into elements of the "risk environment" and how they may influence drug use, fall squarely within the realm of social epidemiologic research, 
as attempts to understand how context shapes the risk of drug dependence and its consequences.

This recent growth in social epidemiologic research is driven largely by four factors (Kaplan, 2004). First, there is an abiding interest in population health sciences about how social factors influence health and health behaviour. Second, there has been a growing awareness of social inequalities in health leading to efforts that aim to understand these inequalities for the purposes of eliminating them. Third, the widespread adoption of multilevel modelling methods has made it possible to simultaneously incorporate characteristics of individuals and of their community or context in epidemiologic analyses (Diez Roux, 2002; O'Campo, 2003). Fourth, in the area of health behaviour research, social epidemiologic inquiry has in many respects formalized the study of factors that have long been suspected to be important determinants of health behaviours. Nearly all conceptual models of health behaviour recognise that an individual does not exist separately from her/his context. In the context of drug use behaviour, social epidemiologic observations about the influence of environmental factors in drug use behaviour have, in many respects, helped empiricize long-standing observations about the social nature of drug use and about the inextricable link between context and drug use behaviour (Zinberg, 1986). A social epidemiologic approach provides a way of formalizing this observation and provides useful guidance for analyses focused on understanding the production of health behaviour.

While the cumulative body of social epidemiologic work has provided us with some interesting insights about specific correlates of health behaviour, perhaps more importantly, social epidemiologic inquiry has helped move us towards an understanding that factors at multiple "levels" influence the production of health and disease. This thinking, referred to as "multilevel" thinking in the peer-reviewed literature (Diez-Roux, 2000; Kaplan, Everson, \& Lynch, 2000), has enabled us to consider, both conceptually and empirically, how characteristics of individuals, their social networks, characteristics of where they live (ranging from neighbourhoods to countries of residence) are the pathways that contribute, individually and jointly, to health and health behaviour. Therefore, a social epidemiologic perspective has provided us an opportunity to consider a "missing piece" of deterministic thinking about health behaviour that traditionally focuses only on features of individuals as determinants of individual behaviour (Baker, Metzler, \& Galea, 2005).

There is little doubt that multilevel thinking has "arrived" in public health research in general, and in drug use research in particular. For example, different authors have used multilevel methods to assess, among many others, the role of the urban built environment as a determinant of alcohol use behaviour (Bernstein, Galea, Ahern, Tracy, \& Vlahov, 2007), the link between neighbourhood socioeconomic status and heroin and cocaine use (Williams \& Latkins, 2007), neighbourhood effects on drug program treatment efficacy (Yabiku et al., 2007) and the relation between neighbourhood income inequality and drug overdose related mortality (Galea et al., 2003). These peer-reviewed publications and many other recent papers considering multilevel questions make use of "multilevel models," which are regression models that computationally can account for the clustered structure of multilevel data (DiezRoux, 2000). Multilevel models allow the estimation of the relation between exposure and outcome of interest while controlling for covariates at different levels and the estimation of variation in the effect of the key exposures across levels of other variables. For example, a multilevel model can assess the relation between the quality of the neighbourhood built environment and likelihood of drug use activity while controlling for the differences between neighbourhoods in individual race/ethnicity and educa- tion (Hembree et al., 2005). Therefore, multilevel models represent an opportunity to quantify the determinants of health across levels, isolate characteristics of an individual's context (families, neighbourhoods, cities, states, or countries) that are associated with individual health behaviour and, in theory, provide guidance for evidence-based interventions targeting contextual factors as well as individual ones.

However, as social epidemiologic inquiry matures and research focuses increasingly on challenging questions of multilevel causation, it is becoming clear that there are substantial conceptual challenges that we face when trying to understand health behaviours using the dominant epidemiologic analytic paradigm (Diez-Roux, 2007; Galea \& Kaplan, submitted for publication). Epidemiologic inquiry is predicated on the notion that we are studying the "determinants" of health and disease states. This central formulation suggests that we are looking to isolate "causes" that unidirectionally influence the disease states of interest. In many respects this focus is understandable since epidemiologic inquiry arose out of clinical medicine where the disease state is the outcome of central concern and where all else focuses on identifying determinants of that outcome. However, it is unlikely that risk factors act independently and unidirectionally to produce health outcomes in general, and in particular health behaviours of any kind. Rather, it is much more plausible that personal circumstances and behaviours that have traditionally been considered "risk factors", are interdependent, shape one another, and are in turn shaped by health and health behaviours. To take but one example, while it may well be the case that features of the urban built environment are associated with greater exposure to stress and consequent drug use behaviour to alleviate that stress, it is also likely that drug use behaviour contributes to more limited economic activity in any given neighbourhood, which in turn may be associated with neighbourhood deterioration and a worse urban built environment both of which contribute to the push and pull of different subpopulations in particular neighbourhoods.

The dominant epidemiologic methodologic approaches rest on the use of various types of regression models - whether across multiple levels or within single levels of interest - to assess the relation between "independent" variables and "outcome" variables of interest in the population health sciences. In many respects this has served epidemiology well. Regression models allow data-driven explanation of the relation between "exposure" and "outcomes", while taking into account multiple confounders that bedevil epidemiologic inquiry.

However, regression models do not allow us to take into account the inter-relations, reciprocity, or discontinuous nature of the relations that likely underlie the determination of behaviour in the "real world". Nor, do they properly recognize the pathways that link various levels of determinants. Recognizing this complexity and interdependence requires the use of methods that move beyond observational deterministic models, and that allow us to take into account the interrelated, dynamic factors across different levels of influence shape health behaviour. One promising avenue in this vein is complex systems computational modelling.

\section{The potential of complex systems modelling approaches}

Complex systems modelling approaches have the potential to integrate our growing knowledge about multilevel determinants of population health, patterns of feedback and interaction between determinants at different levels, and to inform our knowledge about how specific policy interventions influence the pathways that shape the health of populations. For the purpose of this paper, we define complex systems approaches as computational 
approaches that make use of computer-based algorithms to model dynamic interactions between individuals within and across levels of influence using simulated populations. Complex systems analytic approaches have been embraced and used extensively in many other disciplines (Agar, 2005; Agar \& Wilson, 2002; Caulkins, Behrens, Knoll, Tragler, \& Zuba, 2004; Caulkins, Dietze, \& Ritter, 2007; Dray, Mazerolle, Perez, \& Ritter, 2008; Flynn, 2000; Kahan, Rydell, \& Setear, 1995; Kahan, Setear, Bitzinger, Coleman, \& Feinleib, 1992; Levin, Roberts, \& Hirsch, 1972; Levin, Roberts, \& Hirsch, 1975; Perez \& Dray, 2005; Perez et al., 2006). The example established by these other disciplines has the potential to suggest methods and approaches that can be immensely useful to our understanding of health behaviour. For example, economics and sociology have considered both the joint characteristics of individuals and of global societal dynamics that influence economic systems (Lansing, 2003; Tesfatsion, 2002). Economists have adopted complex systems analytic approaches including evolutionary complex models that take into account competing trading strategies. In turn, these strategies can explain observed market behaviours such as clustered volatility (Hommes, 2002) or abrupt changes in short-term trading behaviour (LeBaron, 2002). Complex systems computational approaches also have been applied in organisational science using multi-agent approaches to model realistic organisational behaviour and have direct applicability for policies aimed at improving organisational effectiveness (Carley, 2002). In political sciences, complex systems computational models have been applied to questions of state formation, power politics (Cederman, 1994, 2002) and the role of power sharing in encouraging secessionism (Lustick, Miodownik, \& Eidelson, 2004).

Importantly, although some work in complex systems analytic approaches remains highly theoretical and primarily focused on the modeling exercise per se, other uses are grounded in the use of real data. For example, Axtell et al. (2002) used agent based modelling to model population growth and collapse of the Kayenta Anasazi in Long House Valley between 1800 BCE and 1300 CE. This particular approach used archeological data to provide inputs to a multi-computational model of the society of interest. Results from the computational model were able to reproduce the main features of the known archeological record and to suggest possible explanations for the rapid population decline of the Anasanzi. Complex systems models of civil violence have been shown to parallel observed scenarios and have helped inform our understanding of how group behaviour may lead to communal violence (Epstein, 1999).

Social sciences in general and population health sciences in particular have lagged substantially behind other disciplines in the adoption of these approaches. Importantly, although a few key authors have been advancing the application of complex systems methods in sociology (Macy \& Sato, 2002), we are not aware of comparable progress in population health sciences.

Although we, and others, have called for a growing integration of complex systems methods into public health analysis (Auchincloss \& Diez Roux, 2008; Galea, Ahern, \& Karpati, 2005; Kaplan, 2004; Koopman \& Lynch, 1999), we are aware of only a handful of applications of complex systems computational approaches to population health sciences or health behaviour studies (Levy, Nikolayev, \& Mumford, 2005). The field of infectious disease transmission is an exception as complex systems methods are increasingly being used to model person-to-person transmission of disease in populations.

It has been aptly suggested that the use of complex systems methodology is "reason for optimism" as it may allow analysts to answer a set of research questions regarding systems with large numbers of individuals whose patterns are not easily predictable by an assessment of these individuals alone (Johnson, 1999). We suggest that health behaviour research is an obvious candidate for the application of complex systems modelling approaches that may help us address empiric questions not otherwise answerable using the regression approaches that are commonly applied in the field. There are several features of this research that suggest suitability of complex systems models to the understanding of health (Epstein, 1999) and compelling reasons why complexity modelling approaches may be applicable to questions pertaining to health behaviour (Henrickson \& McKelvey, 2002), particularly within a social epidemiologic framework.

First, health behaviour research is concerned with questions regarding health among heterogeneous individuals. Individual heterogeneity poses a substantial challenge to regression modelling techniques in that it is frequently difficult to genuinely or convincingly "hold constant" all other individual characteristics of interest while determining specific parameters of association that explain relations between individual characteristics. Complex systems approaches allow us to explicitly introduce dynamic relations between individuals and to vary characteristics of heterogeneous individuals within population systems (Bonabeau, 2002).

Second, although individuals may be autonomous, social factors are by definition relational, arising from the interaction between individuals. Therefore, a full understanding of the relative contribution of individual factors and social factors to health behaviour must take into account both individual autonomous action and social interrelations. These interrelations are not usefully summarized using aggregate modelling techniques but are a central feature of complex systems computational techniques.

Third, individual behaviours within population systems are informed both by explicit spatial interaction among individuals and also by the characteristics of the physical space within which individuals reside and other spaces with which the individuals might interact. Despite the enthusiasm for multilevel modelling in recent years, multilevel models remain predicated on the limitations and assumptions of regression techniques and, as such, cannot account for complex and dynamic interactions between individuals within a space and between individuals and features of the space where they reside. In addition, the ultimate aim of multilevel models is to partition variance or estimate effect sizes at different levels, thereby missing the dynamic interactions that may occur between levels. In contrast, complex systems modelling approaches would consider the dynamic consequences of changes at one level on other levels.

Fourth, increasingly we are recognizing that health behaviour is determined both by individual factors and by population factors that have no individual analog. Insofar as health behaviour is determined by factors and conditions at multiple levels of influence, complex systems methods may offer insights into how such micro-level interactions and micro-, meso-, and macro-level factors produce observed health behaviours (Fearon, 1996; Johnson, 1999). These complications have been well documented in classic infectious-disease models where it has been shown that we cannot use regression techniques and that even mildly complicated models are difficult (if not impossible) to analyse mathematically (Koopman, 2003).

Ultimately, we suggest that the reasons discussed here will inevitably result in growing interest in the application of complexity approaches to health behaviour research in coming years. Insofar as health behaviour is predicated on dynamic interaction of factors at multiple levels of influence within human systems, these approaches will be essential for us to enhance our understanding of the determination of health behaviour and subsequently guide intervention to improve the health of populations (Koopman \& Lynch, 1999; Mitchell, 1999). 


\section{An illustration}

In order to illustrate the potential of complex system dynamic models applied to public health, we present here one particular analytic example, a "toy model" essentially, that illustrates a way in which we may consider how attributes of individuals, their interactions, and the space within which the individuals "live" shape individual drug use behaviour. We developed for this illustration one particular type of complex system model-an agent-based model (Gilbert, 2007), a flexible approach to implementing complex system approaches to population health questions. Our model was designed to assess how characteristics of persons who are selling drugs (here referred to as "drug sellers") interrelate with individual likelihood to use drugs in shaping drug dependence. In particular through use of an agent-based model we were able to explore how varying characteristics of an agent's ("individual's") risk environment, including the influence of her/his network and her/his likelihood of being influenced by others who are like her/him result in different dependence probabilities at the model steady state.

We implemented our model as an agent-based model using the REPAST software. Agent-based models allow the simulated creation of persons, typically referred to as "agents", each one of which is autonomous, possesses certain attributes, and may behave differently than all other agents in the modelling environment. Therefore, in model construction, both agents and their attributes, and the physical space they occupy are specified. Once the agents are constructed and placed in space, the model simulates the passage of time by incrementing a clock in discrete steps, and at each time step each agent updates their own internal state based on programmed rules and feedback from the environment.

In our simulated model agents are each endowed with two static attributes: shape and colour. We note that, of course, we mean these characteristics to be exemplars. In a fully parametrized model (i.e., one that is informed by characteristics of populations, based on observational or experimental data) these characteristics may represent, for example, individual race/ethnicity and gender. In addition to these two basic attributes, each agent has a static list of friends, and we call the graph whose vertices are agents and edges are friendships "the social network." We will describe how to construct this graph below, after we describe the space agents occupy.

The space the agents occupy is a $120 \times 120$ rectangular grid that we further subdivide into an $8 \times 8$ grid of $15 \times 15$ blocks. Therefore there are a total of 64 blocks, each with 207 agents [each grid has fewer than maximal (225) number of agents, since there are, as discussed below, some empty spaces for sellers], for a total of 13,248 agents. Once again, these grids can, in fully parametrized models, represent spaces of interest with particular shapes, including, for example, neighbourhoods of residence that may have characteristics that influence the health behaviour in question.

We create a model where the agents with different attributes (colour and shape) are partially segregated in a physical grid space. Therefore, we specify that certain blocks are round-only, greenonly, or round-and-green. We assigned agents to each block so that every block had the same number of agents and in four distinct areas of the model space and each block had agents with a given pair of attributes. For example, in the northwest $2 \times 2$ grid of blocks there are 828 round-green agents, while in the southeast $6 \times 6$ grid of blocks there are 1656 square-green agents, 2484 roundblue agents, and 3312 square-blue agents. In each block there are 18 unoccupied squares.

We construct the social network by specifying, for simplicity, that each agent is friend with their immediate neighbours; most agents have four, but those on the edge of space will have fewer. The resulting graph exhibits only one of two properties of a so-called small-world graph (Newman \& Watts, 1999; Watts \& Strogatz, 1998) wherein two of an agent's friends are much more likely to be friends of each other than a randomly selected pair of agents. By adding edges for several randomly chosen pairs of agents, we can force the social network to have the other key property of a small-world graph: on average, there is a "short" path connecting a randomly chosen pair of nodes.

After placing agents in space we distribute drug sellers in unoccupied squares using a two-tiered approach. First, for each block we specify that half of the blocks are seller free. More precisely, we chose one of the two possible arrangements where two blocks with sellers or two blocks without sellers are never adjacent (i.e. as squares on a chessboard). Next, for those blocks that are not seller free, we specify that each unoccupied square contains a seller with a probability computed as follows. We look at the $5 \times 5$ grid of squares centred on a given unoccupied square and for each of the four single attribute values we calculate the weighted number of agents in the grid with matching value; increasing the size of the grid or weakening the dependence of the weight on distance will smooth out stochastic variations in the weighted number.

During each time step of the model, an agent has a probability of having access to drugs that is a function of the distance to nearby sellers. The probability an agent is addicted at time $t$ depends on three things: whether or not they have access to drugs, their addiction at time $t-1$, and the addiction of their friends at time $t-1$. We are mainly interested in how the average incidence rate of dependence varies with time when we vary each of the key parameters of interest.

We describe here three different scenarios in which we fix all but one of these parameters and let the remaining parameter vary over a limited range. In each scenario we present two graphs. All graphs represent the key variable of interest here (drug dependence) on the $y$-axis as a function of varying one other characteristic at a time. In the first of each pair of graphs we computed the dependence rates for each of the individual attributes (shape and colour), so that everyone was counted twice, and in the second of each pair of graphs we computed the rates for each of the four combinations of shape and colour, so that everyone was counted once. All graphs are shown in Fig. 1.

Example 1. What is the effect of an increase in network influence on population drug dependence rates?

In Example 1, we vary $v$ - the network influence. We can see that initially, increasing $v$ causes the average dependence in some groups to increase and in other groups to decrease, but for larger values of $v$, every group experiences a decrease. The relative differences in dependence rates for all values of $v$ arise from the different conditional probabilities of having access to drugs each group experiences; these probabilities vary due to colour- and shape-based segregation. For example, sellers are most likely in square-blue neighbourhoods hence dependence rates are highest in these two groups of agents. For all groups, dependence rates decrease for large values of $v$ because the majority behaviour of the population starts to override any individual tendencies, and the majority of people have limited access to drugs and a minority of friends who are addicted. That is, for large values of $v$ everyone conforms to the majority behaviour, which favours non-dependence in this case. For in-between values of $v$, one finds cohesion among small groups but not the entire population, so addictive behaviour is reinforced for those groups who have easy access to drugs while non-addictive behaviour is reinforced for those groups who do not have easy access. This then manifests as an increase in dependence rates for higher early values of network influence among the square and blue groups in contrast to a monotonic decrease in dependence 
(a) Example 1.
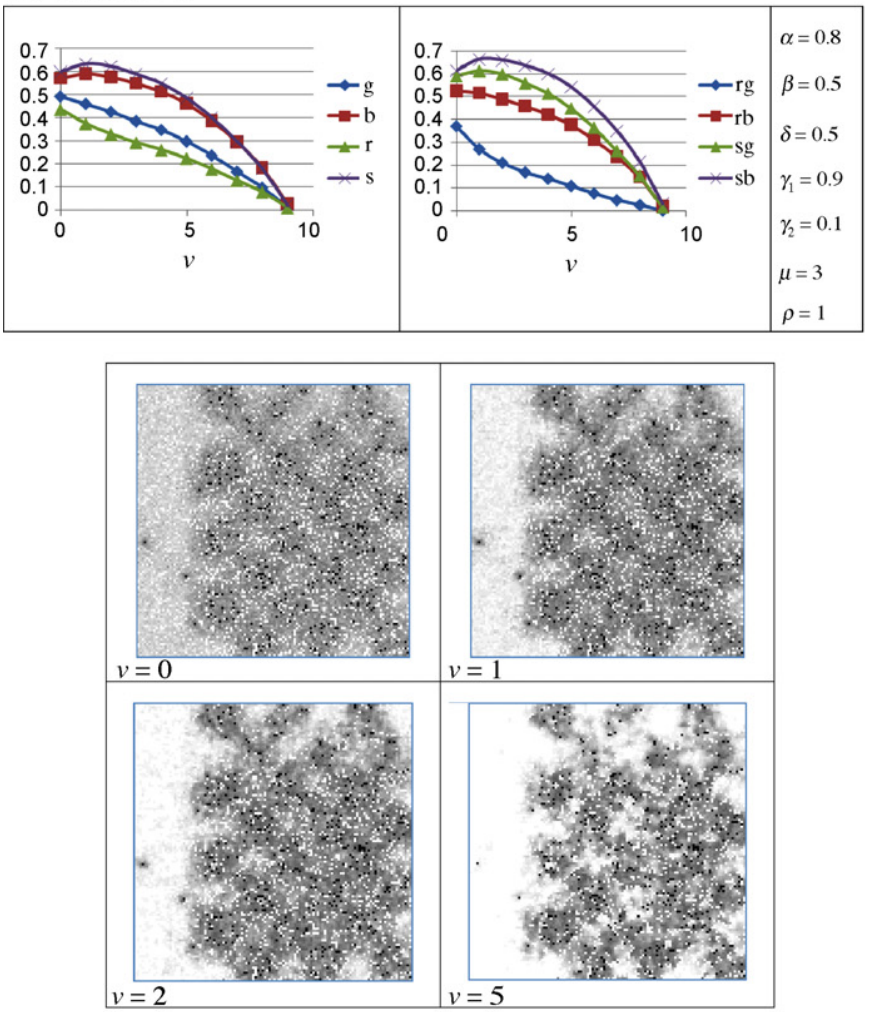

(b) Example 2.
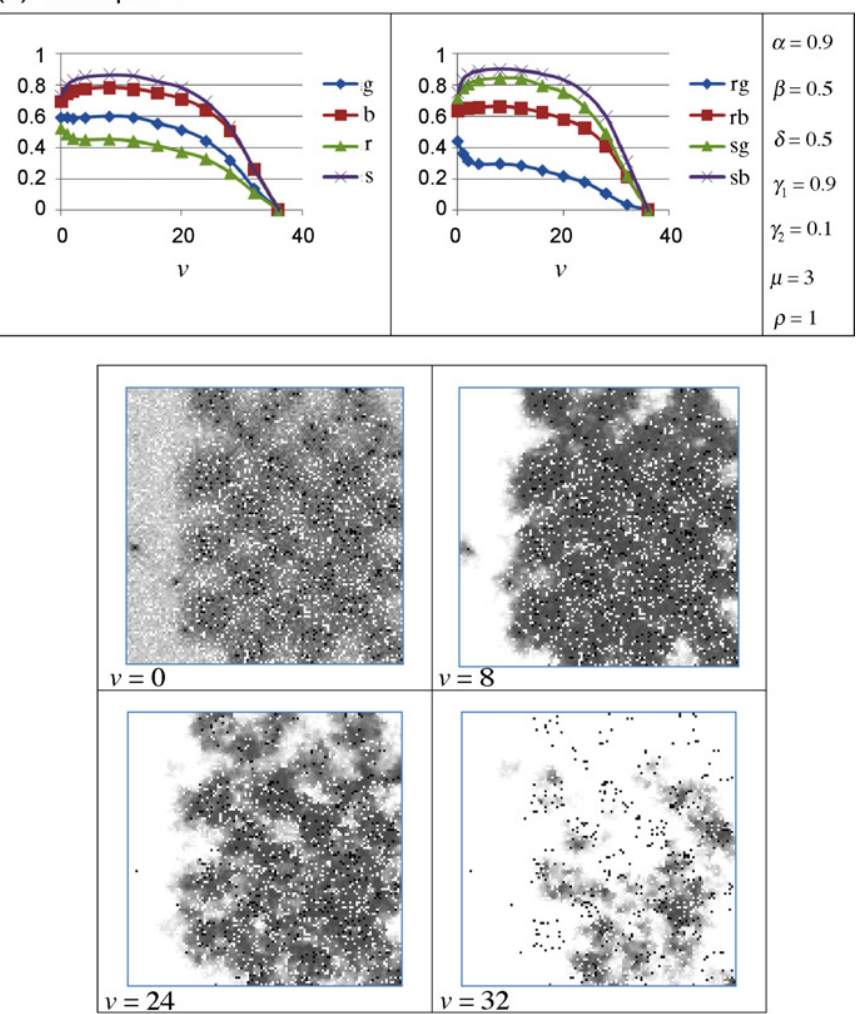

(c) Example 3
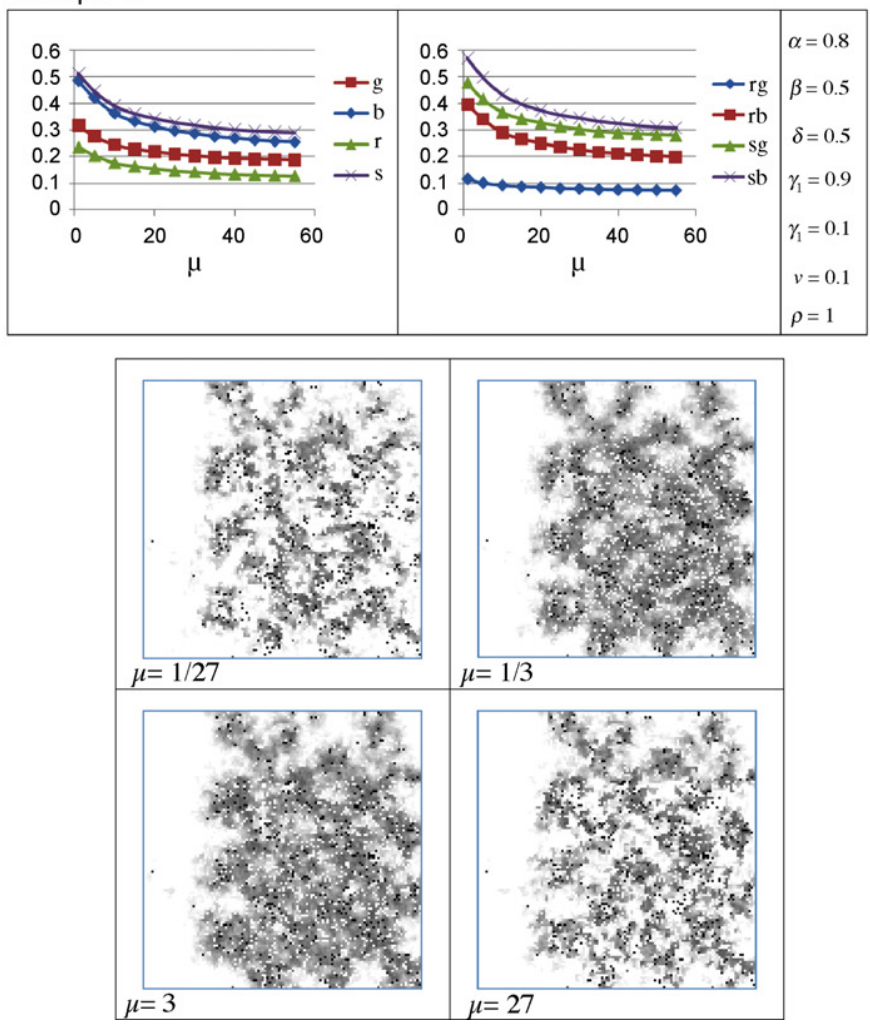

Fig. 1. Modeled scenarios varying different properties of an agent-based model universe predicting drug dependence holding other characteristics of modeled universe constant. The line graphs show population prevalence of drug dependence for the different groups of interest. The two-dimensional figures show the simulated population within a particular physical space, with the modeled agents who are drug dependent being in the darker colour and the non-drug dependent in the lighter colour. The black dots represent drug "dealers", or availability of drugs. 
rates with increasing levels of network influence for the round and green groups. Note, that even for large values of $v$ one can find small pockets of people with high dependence rates, and not surprisingly they centre on areas with a high density of sellers. Also, these pockets can grow and shrink over time and even move around the city, though they are very rare in areas with a low density of sellers, so they tend to die out once they enter areas with relatively few sellers.

Example 2. Does the network influence on drug dependence rates vary by characteristics of the drug itself?

In Example 2, the scenario is similar to the previous one, except that we have now increased the addictiveness of the drug. One important difference in outcomes is that now the network-influence parameter $v$ must be much larger before drugdependence rates drop to zero. The other important difference is that the largest dependence rate in this scenario is bigger than in the previous $(0.85$ versus 0.65$)$. One subtler difference is that the green and round-green groups experience a small increase in dependence rates for small values of $v$, whereas in the previous scenario the dependence rates always dropped as $v$ increased. It is also interesting to note that there is a range of values for $v$ (roughly $4 \leq v \leq 16$ ) where the dependence rates do not vary.

Example 3. Does it matter for drug dependence rates if individuals are more influenced by different types of networks (i.e., network heterogeneity)?

In Example 3, we vary the influence that each of an agent's friends has as a function of whether or not they have the same colour or shape. For values of $\mu$ close to 1 , there will be little difference in the influence as a function of shape and colour. For values of $\mu$ close to 0 , an individual will be more influenced by agents who are different in at least one attribute, and more strongly influenced by those who differ in both attributes. Similarly, for large values of $\mu$, having one attribute in common increases influence, and having two in common increases it further. Increasing $\mu$ has the most pronounced effect for square blues because they tend to have fewer friends with one or both attributes in common, while round greens tend to have more friends with at least one common attribute, so increasing $\mu$ has the smallest effect for them.

\section{Conclusions and research directions}

Using a simple agent-based model that simultaneously considers several factors that may influence drug dependence rates in a particular community we illustrate several observations that reinforce the need for both a social epidemiologic perspective in health behaviour research and show how the application of complex system computational models can help realise some of the promise of this perspective. First, we show here that highly plausible features of the risk environment - including features of an individual's network and of the addictiveness of drugs available - are likely to influence rates of drug dependence and any attempt at understanding drug dependence that does not take these factors into account is likely to be insufficient at best, and misleading at worst. This suggests that a social epidemiologic perspective that takes into account factors at multiple levels of influence within a dynamic framework may be necessary to help us understand health behaviour.

Second, we show here how even in this very simple toy model, the parameters of interest realistically interrelate in such a way that our traditional analytic tools, namely unidirectional "cause-effect" models, are mathematically incapable of dealing with.

Third, an agent-based model allows us to observe particular group-dependence behaviours that illuminate key inputs that influence drug dependence in this population. For example, we observe that different levels of social network influence have different effects on drug dependence rates in different groups; importantly, in groups which have easy access to drugs more network influence is associated with more dependence and the converse is true in groups with less access to drugs (Example 1 ). This empiric observation mirrors one in a very recent paper that showed the interactive effect of collective efficacy and social norms in shaping population smoking risk (Ahern, Galea, Hubbard, Midanik, \& Syme, 2008). There are several other observations that emerge from this model. In Example 2, we can study the competing contributions of drug characteristics and network influence on rates of drug dependence-in the case of highly addictive drugs, network influence matters much less than in the case of less addictive drugs. These observations have clear policy and intervention implications.

Recognizing that social networks are part of the risk environment that may predispose a person to drug dependence does not allow the nuanced recognition of the differential role that may be played by social networks for drugs with different addictiveness (as is shown through comparing Examples 1 and 2). Similarly, as we can see in both Examples 1 and 2, network influence may be associated with both increasing (at low levels of influence), and decreasing (at higher levels of influence) rates of population drug dependence, thus suggesting that interventions that aim to influence network risk environments need to take into account the relevant network density to be genuinely informative for intervention purposes. Example 3 then illustrates how network characteristics, here illustrated as influence by agents of different types, further shapes population drug dependenced rates suggesting that interventions aimed at influencing the network risk environment need to account for the different network influence, coincident with varying degrees of drug dependence and with patterns of network homophily.

So what is the relation between these observations and policy or intervention efforts that aim to improve health behaviour generally or minimize drug use more specifically? Policy makers must almost always rely on imperfect information to make decisions to guide their actions. A social epidemiologic perspective brings a broader lens to the study of health behaviour, and encourages us to take into account factors at multiple levels that may influence health behaviour. However, while this broader conception holds promise, it quickly runs into the limits of our typically used methods. Complex system approaches allow us to use computer-based simulations to model different scenarios, where evidence from randomized trials is not possible, informed by our understanding of the determinants of the health behaviour in question, and to attempt to understand how each of the determinants at multiple levels may influence health behaviours. Going back to our illustrative example, the different role that network influence plays in shaping drug dependence given different degrees of drug availability and type of drug (addictiveness) suggest that a "one size fits all" social network interventions may not be optimal.

While this example illustrates some of the promise inherent in this approach, it also leads us to the challenges that lie ahead. The illustrative example we have used here is simply that-illustrative. We made here no effort to accurately parameterize (or "dock") our model with real world numbers that describe the relations between these variables that are included in the model. Therefore, for example, while we show that the role of social network influence differs by a four-fold factor in the context of drugs with different degrees of addictiveness (Examples 1 and 2), a policy maker will not find these examples particularly useful in the absence of clear guidance about the addictiveness of a particular drug.

As we broaden our lens to take into account determinants at multiple levels of influence, the challenge to accurately quantify the influence of each of these determinants grows. While these methods allow us to better model the interrelation among multiple 
determinants, more accurately representing reality, they are only as useful as the inputs used to inform them are accurate and reliable. In many respects, a call for greater use of complex system models to understand social processes is also a call for greater availability of data that accurately quantifies the relations that are embedded in these models. Paradoxically, the attempt to better characterize the interrelations between factors that shape health behaviour may itself lead to the development of studies that aim to more carefully help quantify one "piece of the puzzle". The development of complex system models, as in the one described here, forces the analyst to think carefully and precisely about the interrelations of interest and in many respects makes the relations for which we have limited data abundantly clear.

We suggest that a way forward, building on the observations drawn in this paper, must involve movement on public health researchers and practitioners alike on multiple fronts. First, our conceptualisation of health behaviour needs to adopt a social epidemiologic perspective, taking into account factors at multiple levels that may shape the health behaviour of interest. Second, we need to make the development of models that attempt to accurately represent the dynamic interrelations of interest a central part of our analytic approach in the field. Third, we need to develop studies that are aimed at accurately parameterizing aspects of complex relations that are critical inputs to model behaviour and which may play a key role in determining a particular course of action aimed at improving health behaviour. This work could lead to models that build directly on epidemiologic observational studies and that can provide empiric estimates that are useful to guide policy explicitly. Fourth, those of us concerned with the health of populations and health behaviour must more comfortably adopt a simulation approach to our analyses that, together with our now familiar linear deterministic approach, can go a long way towards helping us understand the full range of determinants of health behaviour and guide where we may intervene to improve population health.

\section{Conflict of interest}

All authors declare that they have no financial and personal relationships with other people or organisations that could inappropriately influence their work.

\section{Acknowledgements}

We would like to thank Jenna Johnson for work with manuscript preparation. This work was funded in part by a Health Policy Investigator Award from the Robert Wood Johnson Foundation (to SG and GK) and by grants from the National Institutes of Health (DA 017642; DA 022123; HD047861).

\section{References}

Agar, M. (July 14, 2005). Agents in living color: Towards emic agent-based models. Journal of Artificial Societies and Social Simulation, 8(1), 2008.

Agar, M., \& Wilson, D. (2002). DrugMart: Heroin epidemics as complex adaptive systems. Complexity, 7(5), 44-52.

Ahern, J., Galea, S., Hubbard, A., Midanik, L., \& Syme, L. S. (2008). Culture of drinking" and individual problems with alcohol use. American Journal of Epidemiology, 167(9), 1041-1049.

Auchincloss, A. H., \& Diez Roux, A. V. (2008). A new tool for epidemiology: The usefulness of dynamic-agent models in understanding place effects on health. American Journal of Epidemiology, 168(1), 1-8.

Axtell, R. L., Epstein, J. M., Dean, J. S., Gumerman, G. J., Swedlund, A. C., Harburger, J., et al. (2002). Population growth and collapse in a multiagent model of the kayenta anasazi in long house valley. Proceedings of the National Academy of Sciences of the United States of America, 99(Suppl 3), 7275-7279.

Baker, E. A., Metzler, M. M., \& Galea, S. (2005). Addressing social determinants of health inequities: Learning from doing. American Journal of Public Health, 95(4), 553-555.
Bernstein, K. T., Galea, S., Ahern, J., Tracy, M., \& Vlahov, D. (2007). The built environment and alcohol consumption in urban neighborhoods. Drug and Alcohol Dependence, 91(2-3), 244-252.

Bonabeau, E. (2002). Agent-based modeling: Methods and techniques for simulating human systems. Proceedings of the National Academy of Sciences of the United States of America, 99(Suppl 3), 7280-7287.

Carley, K. M. (2002). Computational organization science: A new frontier. Proceedings of the National Academy of Sciences of the United States of America, 99(Suppl 3), 7257-7262.

Caulkins, J. P., Behrens, D. A., Knoll, C., Tragler, G., \& Zuba, D. (2004). Modeling dynamic trajectories of initiation and demand: The case of the US cocaine epidemic. Health Care Management Science, 7(4), 319-329.

Caulkins, J. P., Dietze, P., \& Ritter, A. (2007). Dynamic compartmental model of trends in Australian drug use. Health Care Management Science, 10(2), 151-162.

Cederman, L. E. (1994). Emergent polarity: Analyzing state-formation and power politics. International Studies Quarterly, 38(4), 501-532.

Cederman, L. E. (2002). Endogenizing geopolitical boundaries with agent-based modeling. Proceedings of the National Academy of Sciences of the United States of America, 99(Suppl 3), 7296-7303.

Cooper, H. L., Friedman, S. R., Tempalski, B., \& Friedman, R. (2007). Residential segregation and injection drug use prevalence among black adults in US metropolitan areas. American Journal of Public Health, 97(2), 344-352.

Cummings, K. M., Becker, M. H., \& Maile, M. C. (1980). Bringing the models together: An empirical approach to combining variables used to explain health actions. Journal of Behavioral Medicine, 3(2), 123-145.

Diez-Roux, A. V. (2000). Multilevel analysis in public health research. Annual Review of Public Health, 21, 171-192.

Diez Roux, A. V. (2002). Invited commentary: Places, people, and health. American Journal of Epidemiology, 155(6), 516-519.

Diez-Roux, A. V. (2007). Integrating social and biologic factors in health research: A systems view. Annals of Epidemiology, 17(7), 569-574.

Dray, A., Mazerolle, L., Perez, P., \& Ritter, A. (2008). Drug law enforcement in an agentbased model: Simulating the disruption to street-level drug markets. In L. Liu \& J. Eck (Eds.), Artificial crime analysis systems: Using computing simulations and geographic information systems (pp. 352-371). Hershey, PA: Information Science Reference Publisher.

Epstein, J. M. (1999). Agent-based computational models and generative social science. Complexity, 4(5), 41-60.

Fearon, J. D. (1996). Causes and counterfactuals in social science: Exploring an analogy between cellular automata and historical processes. In P. E. Tetlock \& A. Belkin (Eds.), Counterfactual thought experiments in world politics: Logical, methodological, and psychological perspectives (pp. 39-67). Princeton, NJ: Princeton University Press.

Fishbein, M., Triandis, H. C., Kanfer, F. H., Becker, M., Middlestadt, S. E, \& Eichler, A (2001). Factors influencing behavior and behavior change. In A. S. Baum, T. A. Reveson, \& J. E. Singer (Eds.), Handbook of health psychology (pp. 1-17). Mahwah, New Jersey: Lawrence Erlbaum Associates Publishers.

Flynn, S. E. (2000). Drug trafficking, the international system, and decision-making constraints: A policy-making simulation. International Studies Perspectives, 1(1), $45-55$.

Galea, S., Ahern, J., \& Karpati, A. (2005). A model of underlying socioeconomic vulnerability in human populations: Evidence from variability in population health and implications for public health. Social Science and Medicine, 60(11), 2417-2430.

Galea, S., Ahern, J., Vlahov, D., Coffin, P. O., Fuller, C., Leon, A. C., et al. (2003). Income distribution and risk of fatal drug overdose in New York City neighborhoods. Drug and Alcohol Dependence, 70(2), 139-148.

Galea, S., \& Kaplan, G. A. (submitted for publication). Causal thinking and complex system approaches in epidemiology.

Galea, S., Nandi, A., \& Vlahov, D. (2004). The social epidemiology of substance use. Epidemiologic Reviews, 26(1), 36-52.

Gilbert, N. (2007). Agent-based models in the SAGE quantitative applications in the social sciences series. Thousand Oaks, CA: Sage Publications, Inc.

Glantz, K., Lewis, F. M., \& Rimer, B. K. (Eds.). (1997). Health behavior and health education: Theory, research, and practice. San Francisco, CA: Jossey-Bass, Inc.

Glass, T. A., \& McAtee, M. J. (2006). Behavioral science at the crossroads in public health: Extending horizons, envisioning the future. Social Science and Medicine, 62(7), 1650-1671.

Hembree, C., Galea, S., Ahern, J., Tracy, M., Markham Piper, T., Miller, J., et al. (2005). The urban built environment and overdose mortality in New York City neighborhoods. Health \& Place, 11(2), 147-156.

Henrickson, L., \& McKelvey, B. (2002). Foundations of "new" social science: Institutional legitimacy from philosophy, complexity science, postmodernism, and agent-based modeling. Proceedings of the National Academy of Sciences of the United States of America, 99(Suppl 3), 7288-7295.

Hommes, C. H. (2002). Modeling the stylized facts in finance through simple nonlinear adaptive systems. Proceedings of the National Academy of Sciences of the United States of America, 99(Suppl 3), 7221-7228.

Johnson, P. E. (1999). Simulation modeling in political science. American Behavioral Scientist, 42(10), 1509-1530.

Kahan, J., Rydell, C., \& Setear, J. (1995). A game of urban drug policy. Peace and Conflict: Journal of Peace Psychology, 1(3), 275-290.

Kahan, J., Setear, J., Bitzinger, M., Coleman, S., \& Feinleib, J. (1992). Developing games of local drug policy. Santa Monica, CA: RAND Corporation.

Kaplan, G. A., Everson, S. A., \& Lynch, J. W. (2000). The contribution of social and behavioral research to an understanding of the distribution of disease: A multi- 
level approach. In B. D. Smedley \& S. L. Syme (Eds.), Promoting health: Intervention strategies from social and behavioral research (2nd ed., pp. 31-55). Washington, DC: National Academy Press.

Kaplan, G. A. (2004). What's wrong with social epidemiology, and how can we make it better? Epidemiologic Reviews, 26, 124-135.

Koopman, J. S. (2003). Modeling infection transmission. Annual Review of Public Health, 25, 303-326.

Koopman, J. S., \& Lynch, J. W. (1999). Individual causal models and population system models in epidemiology. American Journal of Public Health, 89(8), 1170-1174.

Lansing, J. S. (2003). Complex adaptive systems. Annual Review of Anthropology, 32 183-204.

LeBaron, B. (2002). Short-memory traders and their impact on group learning in financial markets. Proceedings of the National Academy of Sciences of the United States of America, 99(Suppl 3), 7201-7206.

Levin, G., Roberts, E. B., \& Hirsch, G. B. (1972). Narcotics and the community: A system simulation. American Journal of Public Health, 62(6), 861-873.

Levin, G., Roberts, E. B., \& Hirsch, G. B. (1975). The persistent poppy: A computer-aided search for heroin policy. Cambridge: Ballinger Publishing Company.

Levy, D. T., Nikolayev, L., \& Mumford, E. (2005). Recent trends in smoking and the role of public policies: Results from the SimSmoke tobacco control policy simulation model. Addiction, 100(10), 1526-1536.

Lustick, I. S., Miodownik, D., \& Eidelson, R. J. (2004). Secessionism in multicultura states: Does sharing power prevent or encourage it? American Political Science Review, 98(2), 209-229.

Macy, M. W., \& Sato, Y. (2002). Trust, cooperation, and market formation in the U.S and Japan. Proceedings of the National Academy of Sciences of the United States of America, 99(Suppl 3), 7214-7220.

Mitchell, M. (1999). Can evolution explain how the mind works? A review of the evolutionary psychology debates. Complexity, 4(3), 17-24.
Newman, M. E. J., \& Watts, D. J. (1999). Renormalization group analysis of the smallworld network model. Physics Letters A, 263(4-6), 341-346.

O'Campo, P. (2003). Invited commentary: Advancing theory and methods for multilevel models of residential neighborhoods and health. American Journal of Epidemiology, 157(1), 9-13.

Perez, P., \& Dray, A. (2005). Monograph no. 11: SimDrug: Exploring the complexity of heroin use in Melbourne. Fitzroy: Turning Point Alcohol and Drug Centre: DPMP Monograph Series.

Perez, P., Dray, A., Ritter, A., Dietze, P., Moore, T., \& Mazerolle, L. (2006). SimDrug: A multi-agent system tackling the complexity of illicit drug markets in Australia. In P. Perez \& D. Batten (Eds.), Complex science for a complex world-Exploring human ecosystems with agents (pp. 193-224). Canberra: ANU E Press.

Sussman, S., \& Dent, C. W. (1999). One-year prospective prediction of marijuana use cessation among youth at continuation high schools. Addictive Behaviors, 24(3), 411-417.

Tesfatsion, L. (2002). Economic agents and markets as emergent phenomena. Proceedings of the National Academy of Sciences of the United States of America, 99(Suppl 3), 7191-7192.

Watts, D. J., \& Strogatz, S. H. (1998). Collective dynamics of 'small-world' networks. Nature, 393(6684), 440-442.

Williams, C. T., \& Latkins, C. A. (2007). Neighborhood socioeconomic status, personal network attributes, and use of heroin and cocaine. American Journal of Preventive Medicine, 32(Suppl. 6), S203-211.

Yabiku, S., Kulis, S., Marsiglia, F. F., Lewin, B., Nieri, T., \& Hussaini, S. (2007). Neighborhood effects on the efficacy of a program to prevent youth alcohol use. Substance Use \& Misuse, 42(1), 65-87.

Zinberg, N. E. (1986). Drug, set, and setting: The basis for controlled intoxicant use. New Haven, CT: Yale University Press. 